\title{
Future Research Directions for Innovating Pedagogy
}

\author{
Jeremy Roschelle ${ }^{1(\varpi)}$, Louise Yarnall ${ }^{1}$, Mike Sharples ${ }^{2}$, \\ and Patrick McAndrew ${ }^{2}$ \\ ${ }^{1}$ SRI International, Menlo Park, CA, USA \\ \{jeremy.roschelle, louise.yarnall\}@sri.com \\ 2 The Open University, Milton Keynes, UK \\ \{mike.sharples, iet-director\} @open.ac.uk
}

\begin{abstract}
A series of reports on Innovating Pedagogy were launched in 2012 to look at the trends that show how practitioners may engage in innovation in pedagogy. This paper looks at the latest set of trends, and highlights four 2015 trends that seem particularly rich for researchers to explore in the next five years.
\end{abstract}

Keywords: Pedagogy · Educational technology · Innovation · Learning · Instruction

\section{Introduction}

Innovation is often associated with advances in technology, but approaches that make a profound change to education are usually based not on technology but on innovations in pedagogy for a technology-enabled and mobile world. Since the Innovating Pedagogy annual series was launched in 2012, over 30 different trends have been examined. This paper highlights four for research. Since December, the 2015 report has garnered more than 66,000 downloads from 128 countries. Fourteen researchers from The Open University (UK) and SRI International (US) contributed to the latest report.

\subsection{Practitioner Trends}

The image in Fig. 1, produced by TeachOnline, summarizes the 2015 pedagogical trends for practitioners at a glance. For more detail, the reader may review the full report at www.open.ac.uk/innovating.

\section{Four Promising Trends for Research}

To reflect on the prospective future for learning and teaching in school and beyond, we selected four 2015 pedagogical trends that advance long desired pedagogical goals through the use of new technology: Incidental Learning, Context-based Learning, Embodied Learning, and Analytics of Emotions. Future research should focus on how 


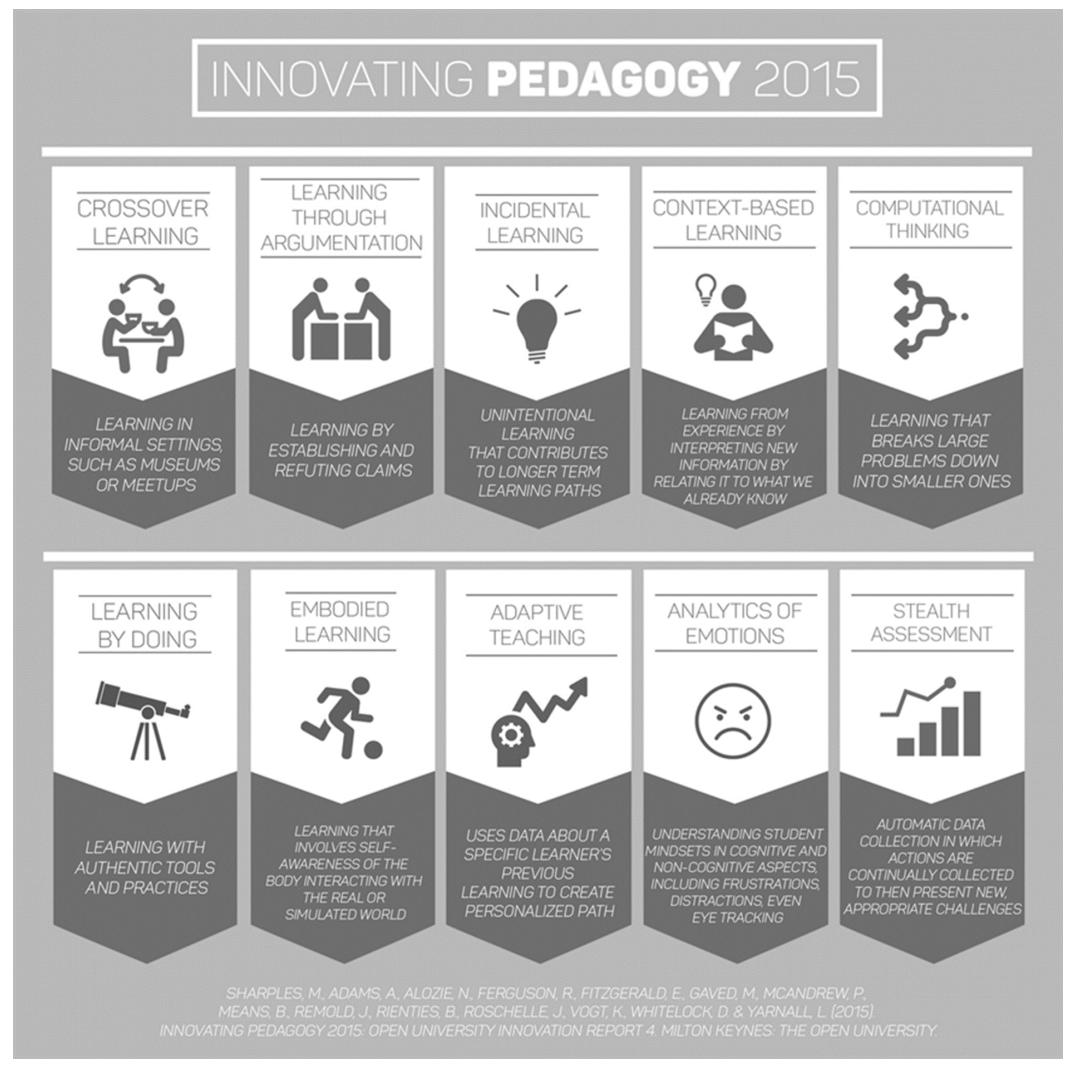

Fig. 1. The 2015 Innovating Pedagogy top 10 trends. Image credit: Stephen Valdivia of TeachOnline, the Arizona State University Instructional Design Community

all four involve intelligent technologies in delivering the most human and powerful features of pedagogy - mentoring, timely information presentation, and responsiveness to the learner's physical and emotional processes.

\subsection{Overview}

Education pioneer John Dewey wrote, "Such happiness as life is capable of comes from the full participation of all our powers in the endeavor to wrest from each changing situations of experience its own full and unique meaning," [1, p. 25]. To begin to wrest meaningful learning from technology-rich situations, we look to the four themes below.

Incidental Learning. Incidental Learning captures learning ephemera for productive use. It brings up pedagogies and technologies for noticing, reflecting, and connecting the unplanned learning that we experience daily. A mobile app may permit a learner to record a feeling or impression after an experience in the workplace, and then later refer back to recall, index, and share it. Learners may receive a text "nudge" to help maintain 
focus on an extended task, thus supporting memory, motivation, planning, revision, and mentoring. Future research drawing on behavioral economics and cognitive behavioral therapy may explore how learners may use technology to record instances of incidental learning so they can reflect on them and obtain social support around them. Theories of social-emotional learning, such as self-determination theory [2], growth mindsets [3], and self-regulation [4], offer a useful starting point for investigation.

Context-Based Learning. Context is both something we are immersed in and something we create. As technology becomes more embedded in life through the so-called Internet of Things, the opportunities for learning in context can be expected to increase. Contextual learning technology may bring realistic simulation into a classroom or project an instructional overlay onto the world through augmented reality in a mobile device. It capitalizes on the human capacity to see similarities and differences when the same process is applied in different settings and conditions. Future research may explore how such technologies can improve knowledge transfer by linking knowledge learned in school with knowledge gained from informal contexts [5]. Research may also examine how learners create context through interaction using technologies [6]. Also useful are theories of knowledge representation, symbol systems, and distributed cognition, particularly for designing augmented reality overlays projected on to an environment. One of the core challenges is to help learners regulate their access to these opportunities and to help educators be aware of when to switch distributed networks on and off as needed.

Embodied Learning. Embodied learning considers how the learner is engaged as a whole person with the learning process. As someone performs a task, new technology can focus attention and help link knowledge to activity, moving the learning from abstract to concrete action that embeds learning deeply. Such embodied learning is not a new concept [7], but technology supports how we can measure performance, as reflected in the use of Fitbits and health apps. Embodied learning presents analytics of both individual and collective activity, permitting comparisons for performance improvement. Future research may focus on designing for embodied experiences, technological transformation, and forms of feedback. This research can cross from neuroscience and educational design to new technologies.

Analytics of Emotions. Analytics of Emotions research identifies the emotions relevant to learning and develops the sensing technologies that can track and respond to learner emotions during online learning. A theme of research since the mid 1990s [8], it is now being extended into classrooms and informal settings. Early work focused on inferring a learner's motivational states from logs of online learning, but more recent studies track states with eye tracking, facial recognition and posture analysis. Such studies aim to help learners understand when they are struggling and need to seek help. This research builds understanding of how emotional constructs interact with attention, memory and understanding. 


\section{Conclusion}

We have discussed the pedagogy of emerging innovation and we invite the research community to consider learning environments that anticipate incidental learning, support an interdependence of content and context, engage the integration of body and mind, and are responsive to learners' emotional states.

Acknowledgements. This material is based in part upon work supported by the National Science Foundation under Grant No. IIS-1233722. Any opinions, findings, and conclusions or recommendations expressed in this material are those of the authors and do not necessarily reflect the views of the National Science Foundation.

Open Access. This chapter is distributed under the terms of the Creative Commons Attribution 4.0 International License (http://creativecommons.org/licenses/by/4.0/), which permits use, duplication, adaptation, distribution and reproduction in any medium or format, as long as you give appropriate credit to the original author(s) and the source, a link is provided to the Creative Commons license and any changes made are indicated.

The images or other third party material in this chapter are included in the work's Creative Commons license, unless indicated otherwise in the credit line; if such material is not included in the work's Creative Commons license and the respective action is not permitted by statutory regulation, users will need to obtain permission from the license holder to duplicate, adapt or reproduce the material.

\section{References}

1. Dewey, J., Hickman, L., Alexander, T.M.: The Essential Dewey: Ethics, Logic, Psychology, vol. 1. Indiana University Press, Bloomington (1998)

2. Ryan, R.M., Deci, E.L.: Self-determination theory and the facilitation of intrinsic motivation, social development, and well-being. Am. Psychol. 55(1), 68-78 (2000)

3. Dweck, C.: Mindset: The New Psychology of Success. Random House, New York (2006)

4. Gollwitzer, P.M., Oettingen, G.: Planning promotes goal striving. Cogn. Physiol. Neurol. Dimens. 2, 162-185 (2011)

5. Salomon, G., Perkins, D.N.: Rocky roads to transfer: rethinking mechanism of a neglected phenomenon. Educ. Psychol. 24(2), 113-142 (1989)

6. Dourish, P.: What do we talk about when we talk about context. Pers. Ubiquitous Comput. 8(1), 19-30 (2004)

7. Lakoff, G., Johnson, M.: Philosophy in the Flesh: The Embodied Mind and Its Challenge to Western Thought. Basic Books, New York (1999)

8. del Solato, T., Du Boulay, B.: Implementation of motivational tactics in tutoring systems. J. Interact. Learn. Res. 6(4), 337-378 (1995) 